\title{
Priming impossible figures in the object decision test: The critical importance of perceived stimulus complexity
}

\author{
MARISA CARRASCO \\ New York University, New York, New York \\ and \\ JOHN G. SEAMON \\ Wesleyan University, Middletown, Connecticut
}

\begin{abstract}
Previous research showed that object decision priming was found for possible, but not impossible, three-dimensional objects (e.g., Schacter, Cooper, \& Delaney, 1990; Schacter, Cooper, Delaney, Peterson, \& Tharan, 1991). We tested those objects and found that the impossible objects were subjectively more complex than the possible objects. We then constructed two sets of possible and impossible objects - one set that was equated for complexity, and one set that differed - for use in the object decision test. The results showed that when impossible objects were high in complexity and possible objects were low in complexity, priming was found only for possible objects; when possible and impossible objects were equated at a moderate level of complexity, priming was observed for both object types. These findings indicate that perceived object complexity, more than object possibility-impossibility, determined priming in the object decision test. The demonstration of object decision priming for possible and impossible objects calls for a reformulation of the structural description system explanation.
\end{abstract}

During the past decade psychologists have devoted considerable effort to distinguishing between explicit and implicit memory. Explicit memory is defined as the conscious or intentional retrieval of past experience, whereas implicit memory refers to the nonconscious or unintentional retrieval of previously acquired information (Graf \& Schacter, 1985; Schacter, 1987). Schacter and Tulving (1994) note that explicit and implicit memory do not represent different types of memory or different memory systems. Instead, these terms characterize different expressions of memory that have been dissociated by a wide variety of experimental variables (see Graf, 1994, Moscovitch, Goshen-Gottstein, \& Vriezen, 1994, Richardson-Klavehn \& Bjork, 1988, Roediger \& McDermott, 1993, and Schacter, 1987, for reviews). The goal of the present study was to examine one particularly robust dissociation that has emerged from this research. Specifically, research by Schacter, Cooper, and their col-

The authors thank Donna Ganor-Stern for assistance in collecting the subjective complexity and impossibility ratings, and Krista Johansen for assistance with data analysis. The authors also thank Ron Kinchla, Richard Marsh, and reviewers Mary Peterson and Paul D'Agostino for helpful comments on an earlier draft of this paper, and J. Gay Snodgrass for helpful comments and the suggestion on reporting measures of relative priming. Correspondence should be addressed to M. Carrasco, Department of Psychology, New York University, 6 Washington Place, 8th Floor, New York, NY 10012-1052 (e-mail: marisa@xp.psych.nyu.edu), or to J. G. Seamon, Department of Psychology, Wesleyan University, Middletown, CT 06459-0408 (email: jseamon@wesleyan.edu). leagues (Cooper, Schacter, Ballesteros, \& Moore, 1992; Schacter \& Cooper, 1993; Schacter, Cooper, \& Delaney, 1990; Schacter, Cooper, Delaney, Peterson, \& Tharan, 1991; Schacter, Cooper, Tharan, \& Rubens, 1991; Schacter, Cooper, \& Treadwell, 1993; Schacter, Cooper, \& Valdiserri, 1992) has consistently shown that people demonstrate above-chance recognition (explicit memory) for possible and impossible objects; however, their research has shown priming in the object decision task (implicit memory) only for possible figures. For the sake of brevity, we will refer to performance on these tasks as reflecting explicit or implicit memory.

In the first of a series of influential papers, Schacter et al. (1990) presented participants with unfamiliar possible and impossible objects in an incidental learning task. After encoding the objects, the participants were given a surprise recognition or object decision test. The recognition test (a measure of explicit memory) required the participants to determine whether each object had been presented during study. The object decision test (a measure of implicit memory) required them to classify each stimulus as a possible or impossible object. Half of the studied and nonstudied stimuli represented possible objects that could exist as three-dimensional forms, and half represented impossible objects that contained surface or edge violations that prevented them from existing as threedimensional structures. Implicit memory was indicated when participants showed priming in the form of greater object classification accuracy for studied objects than for nonstudied objects. Schacter et al. (1990) found that ob- 
ject type dissociated explicit and implicit memory: Recognition was observed for both object types, whereas priming was found for possible figures but not for impossible figures.

Schacter, Cooper, Delaney, et al. (1991) assessed various explanations for the lack of priming for impossible objects. In a modification of their previous procedure, they used only possible and impossible objects for which control participants had shown $95 \%$ or greater interparticipant classification agreement under conditions of unlimited exposure. They also increased the number of exposures to the stimuli at study to enhance the strength of the memory representations, modified the instructions to give more equal weight to possible and impossible decisions, and equated the possible and impossible objects for overall size. None of these manipulations succeeded in producing priming for impossible objects. Schacter, Cooper, Delaney, et al. (1991) concluded that, although priming for impossible objects might yet be observed under different conditions, the "failure to document priming of impossible objects despite numerous experimental variations, indicates that it is unlikely that this finding is attributable to some spurious or idiosyncratic feature of our instructions, materials, or procedures" (p. 16).

Subsequent research by Schacter, Cooper, and colleagues (e.g., Cooper et al., 1992; Schacter \& Cooper, 1993) has provided no additional hypotheses about the putative lack of priming for impossible objects. Ratcliff and McKoon (1995) have addressed this issue according to a response-bias hypothesis, but Schacter and Cooper (1995) have questioned this interpretation. An alternative explanation of the lack of priming for impossible figures remains available: Might this finding be due to systematic differences in figure complexity between possible and impossible objects? While pursuing research on attention and priming with these figures (Ganor-Stern, Seamon, \& Carrasco, 1995), one of us (M.C.) observed that the impossible figures appeared more complex than the possible figures. This observation led to the present study of the effect of stimulus complexity on explicit and implicit memory.

Stimulus complexity has been subjectively and objectively defined. For example, Snodgrass and Vanderwart (1980) asked participants to rate the subjective complexity of common objects according to their amount of detail. Other researchers have defined it objectively by counting the number of points in a random polygon (Attneave, 1957), the number of dots in a dot matrix (Attneave, 1955; French, 1954), and the number of angles, parts, or turns in unfamiliar forms (Chipman, 1977; Fehrer, 1935; Hochberg \& Brooks, 1960). Given that there is no consensus regarding the objective measurement of complexity, in this study we will employ both objective and subjective measures.

Stimulus complexity has not been manipulated in implicit memory research, but there are studies of its effect on explicit memory. Some studies have shown that as objective complexity increases, memory performance de- creases for recall by reproduction (e.g., Attneave, 1955; Murphy \& Hutchinson, 1982) and recognition (e.g., Vanderplas \& Garvin, 1959). Other studies suggest that memory performance may be nonmonotonically related to complexity. Recognition could be enhanced with increases in complexity up to some level, after which it is impaired by further increases (French, 1954), or it may be better for extreme complexity levels than for intermediate levels (Snodgrass \& Vanderwart, 1980). When stimulus complexity is varied multidimensionally, no simple relationship can be predicted.

The goal of the present research was to determine whether object type dissociated explicit and implicit memory under different conditions of stimulus complexity. To accomplish this goal, we used Schacter and Cooper's possible and impossible objects and their general procedures to observe if the lack of priming for impossible objects would still be present when possible and impossible objects were equated for complexity or if it would be present only when the impossible objects were measurably more complex than the possible ones.

\section{PHASE 1}

To determine whether Schacter and Cooper's possible and impossible objects differed in complexity, objective complexity measures and ratings of subjective complexity and possibility-impossibility were obtained for each figure.

\section{Method}

Eighty Wesleyan University students, who received introductory psychology credit, were presented with line drawings of 36 possible and 36 impossible objects provided by Schacter and Cooper (see Schacter et al. 1990, and Schacter, Cooper, Delaney, et al., 1991, for examples). The objects were presented in test booklets for self-paced viewing, and their order of presentation was randomized across participants. Half of the participants rated the objects for complexity, and half rated them for possibility-impossibility on a 7-point scale. For the complexity ratings, 1 meant low complexity and 7 meant high complexity. For the possibility-impossibility ratings, 1 meant highly possible and 7 meant highly impossible. (Although objects are either possible or impossible, participants perceived gradations in the degree of possibility/impossibility of the objects.) In addition, objective measures of complexity were obtained by counting the number of lines, line segments, angles, points, and elements (enclosed areas within a figure) in each object.

\section{Results and Discussion}

Statistical comparisons of the subjective ratings for the possible and impossible objects revealed that possible objects were rated less complex $[t(35)=7.92, p<.001]$ and more possible $[t(35)=26.55, p<.001]$ than impossible objects. A Pearson product-moment correlation showed that the subjective complexity and possibilityimpossibility ratings were highly correlated $[r(70)=$ $.85, p<.001]$, supporting the hypothesis that much of the possibility-impossibility variability could be attributed to the degree of complexity of the figures $\left(R^{2}=72 \%\right)$.

Because Schacter and Cooper have typically used either the same or very similar sets of 20 possible and 20 
impossible objects in their experiments (e.g., Cooper et al., 1992; Schacter, Cooper, Delaney, et al., 1991; Schacter et al., 1993), and the particular stimulus objects that they have used were unknown to us, we obtained random samples of possible and impossible objects from the entire set of 72 objects to determine whether these randomly selected sets would differ in complexity ratings. We selected 10 random samples of 10 possible and 10 impossible objects each and conducted $t$ tests on their complexity ratings. In each instance, the random impossible set was rated more complex than the corresponding possible set (all $p \mathrm{~s}<.0001$ ). However, $t$ tests between pairs of possible sets (10 tests) or between pairs of impossible sets (10 tests) yielded no differences (all $p s>$ .1). In sum, complexity did not differ within possible or impossible sets, but, regardless of the stimuli selected, impossible object sets were rated more complex than possible object sets.

To investigate the relationships between subjective ratings of complexity or possibility-impossibility ratings with objective assessments of complexity, we obtained intercorrelations among these subjective ratings and several objective complexity measures (number of lines, number of segments, number of points, angles, and elements), for five groupings of stimuli to be used in Phase 2: all stimuli (48), equated or extreme stimuli (24 each), and possible or impossible stimuli ( 24 each). In general, impossible objects were perceived as more complex than possible objects. In addition, there were significant positive correlations among all of the objective complexity measures for all groupings of stimuli (all $p \mathrm{~s}<$ .001 ). Subjective ratings of figure complexity increased with increases in objective complexity measures. ${ }^{1}$

Table 1 shows the Pearson correlations obtained between objective measures of complexity and subjective ratings of complexity or possibility-impossibility. Although subjective complexity ratings and subjective impossibility ratings correlated with each other in all groupings, the correlations between objective measures of complexity were more numerous for subjective ratings of complexity than for subjective ratings of impossibility. For all the stimuli, as well as for the groupings by extreme, possible, and impossible, subjective complexity ratings significantly correlated with objective complexity measures. The lack of significant correlations between the objective complexity measures and subjective complexity ratings for the equated stimuli may have been due to the fact that equating complexity compressed the range of values. In contrast, objective complexity measures for these equated stimuli correlated with subjective impossibility ratings, possibly because this was the only condition for which these ratings had a greater range of values than the complexity ratings. Whereas the range of complexity ratings was 3.45-4.50 with a standard deviation of 0.3 , the range for impossibility ratings was $1.45-5.75$ with a standard deviation of 1.6.

Note that subjective ratings of possibility-impossibility may be affected by perceived complexity. Likewise, subjective ratings of complexity may be affected by the degree of perceived possibility-impossibility of the objects. Furthermore, both ratings may also be influenced by other factors, such as the number of depth planes in an object figure or the number of structural violations in an object that define it as impossible. However, the results shown in Table 1 indicate that there is indeed a strong relationship between the measures of objective complexity and the ratings of subjective complexity, and, furthermore, this relationship is much stronger than that between objective complexity measures and subjective impossibility ratings.

\section{PHASE 2}

To determine whether object decision priming could be demonstrated for impossible objects, we replicated the general procedures of Schacter, Cooper, Delaney, et al.'s (1991) Experiment 2 with two sets of possible and impossible objects: an "equated" set in which the possible and impossible objects were matched for subjective complexity, and an "extreme" set in which the impossi-

Table 1

Pearson Correlations Between Objective Measures of Complexity and Subjective Ratings of Complexity or Impossibility

\begin{tabular}{|c|c|c|c|c|c|}
\hline & \multicolumn{5}{|c|}{ Stimuli } \\
\hline & All (48) & Equated (24) & Extreme (24) & Possible (24) & Impossible (24) \\
\hline Complexity $\times$ impossibility & $.814 \ddagger$ & $.449^{*}$ & $.972 \ddagger$ & $.607 \dagger$ & $.549+$ \\
\hline \multicolumn{6}{|l|}{ Complexity $\times$} \\
\hline lines & .129 & .080 & .191 & $.404^{*}$ & $.654 \ddagger$ \\
\hline segments & $.288^{*}$ & .144 & $.413^{*}$ & $.449^{*}$ & $.706 t$ \\
\hline angles & $.366^{*}$ & .211 & $.531 \dagger$ & $.487^{*}$ & $.704 \ddagger$ \\
\hline points & $.279^{*}$ & .100 & $.402 *$ & $.456^{*}$ & $.709 \ddagger$ \\
\hline elements & $.411 \dagger$ & .243 & $.569 \dagger$ & $.508^{*}$ & $.689 \ddagger$ \\
\hline \multicolumn{6}{|l|}{ Impossibility $\times$} \\
\hline lines & $-.304^{*}$ & $-.697 \ddagger$ & .034 & -.168 & .032 \\
\hline segments & -.128 & $-.625 \ddagger$ & .276 & -.072 & .129 \\
\hline angles & -.037 & $-.563 \dagger$ & $.419^{*}$ & -.074 & .182 \\
\hline points & -.154 & $-.671 \ddagger$ & .259 & -.125 & .108 \\
\hline elements & .046 & $-.498^{*}$ & $.475^{*}$ & .060 & .203 \\
\hline
\end{tabular}


ble objects were more complex than the possible objects. If the lack of priming for impossible objects is due to an inability of participants to generate implicit memory representations for these figures, no priming for impossible figures should occur even when they are equated to possible figures for complexity. Alternatively, the observation of priming for impossible figures would imply that the previous failures reported by Schacter, Cooper, and colleagues (e.g., Schacter et al., 1990; Schacter, Cooper, Delaney, et al., 1991) were due to the use of possible and impossible figures that differed systematically in perceived complexity. It is important to note that the extreme complexity relationship could not be reversed for an alternative set of extreme figures because possible objects were never rated more complex than impossible objects.

\section{Method}

Participants and Design. One hundred Wesleyan University students, who received pay or introductory psychology credit, took part in a $2 \times 2 \times 2 \times 2$ mixed factorial design. Object complexity (equated vs. extreme) and test type (object decision vs. recognition) were manipulated as between-subject variables; object type (possible vs. impossible objects) and item type (studied vs. nonstudied objects) were manipulated as within-subject variables. The object complexity and test type variables yielded four experimental groups, each composed of 20 participants. A fifth group of 20 participants served in an object decision control condition.

Complexity ratings from Phase 1 were used to construct two sets of possible and impossible objects that had no figures in common. In one set, 12 possible and 12 impossible objects were selected to be virtually equal in subjective complexity; in the other set, 12 possible and 12 impossible figures were chosen to be maximally dissimilar for this variable. The equated figures had a complexity rating mean of 3.87 (range, 3.45-4.50) for the possible objects and 4.06 (range, 3.63-4.43) for the impossible objects, whereas the extreme figures had a complexity rating mean of 2.52 (range, $2.03-2.85$ ) for the possible objects and 5.32 (range, 4.95-6.00) for the impossible objects. Within the extreme and the equated sets, the studied and nonstudied stimuli were assigned to be maximally similar in terms of subjective complexity ratings.

Subjective complexity and possibility-impossibility were positively correlated for all 48 figures and the 24 extreme figures, but they were also correlated for the 24 equated figures (Table 1). Notwithstanding our efforts to equate the complexity of one set of possible and impossible objects, a correlation of complexity and possibilityimpossibility was still present, although minimized. Finally, because there was only partial overlap in the distributions of complexity ratings for possible and impossible figures, the extreme complexity relationship could not be reversed (i.e., possible objects more complex than impossible objects) for an alternative set of extreme figures.

Stimulus figures were photographed as negative slides and projected on a screen by a Gerbrands projection tachistoscope. All subtended a visual angle of approximately $7^{\circ}$ when viewed from a distance of $2.75 \mathrm{~m}$, and they appeared as red objects on a dark surround when shown through a red photographic filter attached to the lens shutter of the tachistoscope. The experiment was conducted under conditions of low room illumination.

Procedure. The participants were told that they would see a series of 60 three-dimensional objects, and they would have to decide if each object was facing left or right. Two examples were shown to emphasize the subjective aspect of the perceptual judgment. The participants, who were tested in groups of up to 8 , were not informed of the possible impossible stimulus dimension or the subsequent object decision or recognition test.

During study, the participants in the four experimental groups were shown 12 object figures five times each in five random orders of 12 Half of the participants were shown object figures from the complex- ity equated set, and half were shown figures from the complexity extreme set. Within each set, half of the figures were possible objects, and half were impossible objects. Each figure was presented in the center of the visual field and shown for $1 \mathrm{sec}$, followed by a 4-sec interstimulus interval. The participants were instructed to look at each figure for the full exposure duration and then mark their answer sheets if the figure was primarily facing left or right. This structural encoding condition was the same as that used by Schacter et al. (1990; Schacter, Cooper, Delaney, et al., 1991), except that the present participants were given the option of selecting Hard to Tell if they could not determine whether a figure was facing left or right. This response was selected on $9 \%$ of all trials.

Following exposure to the study stimuli, the participants were presented with 48 test trials containing 24 figures from the study portion of the experiment and 24 comparable figures not previously studied. The studied and nonstudied figures were presented individually in a random order. Half of the participants received the object decision test, and half received the recognition test. For each test type, the participants were tested with object figures from the same set of equated or extreme objects as shown during study. The participants given the object decision test were told to classify each test figure as a possible or impossible object by marking their answer sheets accordingly. Prior to the test, they were given detailed instructions about possible and impossible objects, including four possible and four impossible object examples to classify, and they were told that half of the figures to be shown represented possible objects and half represented impossible objects. Alternatively, the participants given the recognition test were told to try to recognize the previously shown objects by making a "yes" or "no" response to each test stimulus on their answer sheets. Each test trial consisted of a verbal "ready" signal, followed by a 100msec (object decision test) or a 3-sec (recognition test) stimulus exposure and a group-paced intertrial interval of approximately 4 sec.

Finally, to be sure that any differences in object decision performance for studied and nonstudied objects were not due to differences in the particular objects designated as studied and nonstudied in the equated and extreme object sets, the participants in the control condition were given the object decision test for both the equated and the extreme object sets without prior study. The test instructions and conditions were the same as those described previously for the object decision test with one exception: 96 trials were presented ( 48 for each object set).

\section{Results and Discussion}

Object decision priming. The results of the control condition showed that classification accuracy did not differ significantly for objects designated as studied or nonstudied for the complexity equated $[F(1,19)=3.32$, $p<.1$; nonstudied objects were classified slightly better than were studied objects] or complexity extreme $(F<1)$ object sets. These data indicate that any differences in object decision performance for studied and nonstudied objects in the experimental conditions were not due to intrinsic differences in the particular studied and nonstudied objects used in the equated and extreme object sets.

For the complexity equated condition, studied objects were classified more accurately than were nonstudied objects $[F(1,19)=34.97, p<.001]$, there was no difference in performance for possible and impossible objects $[F(1,19)=2.81, p>.1]$, and there was no interaction of object and item type $(F<1)$. Performance was better for studied items than for nonstudied items for both possible objects $[F(1,19)=9.05, p<.01]$ and impossible objects $[F(1,19)=4.75, p<.05]$. As shown in Figure 1a, these data provide the first demonstration of priming in the object decision test for both possible and impossible objects.

For the complexity extreme condition, the participants classified studied objects more accurately than nonstud- 
ied objects $[F(1,19)=7.80, p<.02]$ and possible objects more accurately than impossible objects $[F(1,19)=$ $8.39, p<.01]$. There was an interaction of object and item type $[F(1,19)=6.07, p<.03]$, indicating the presence of priming for possible objects $[F(1,19)=10.37$, $p<.001]$ but not impossible objects $(F<1)$.

Furthermore, when relative priming was calculated [(studied - nonstudied $) /(100$ - nonstudied $)]$, we found that the amount of priming was a function of complexity; the less complex the items, the higher the relative priming: extreme possible $=.79 ;$ equated possible $=.44 ;$ equated impossible $=.31 ;$ extreme impossible $=-.11$. Note that the difference between the extreme and equated sets of

a) PRIMING

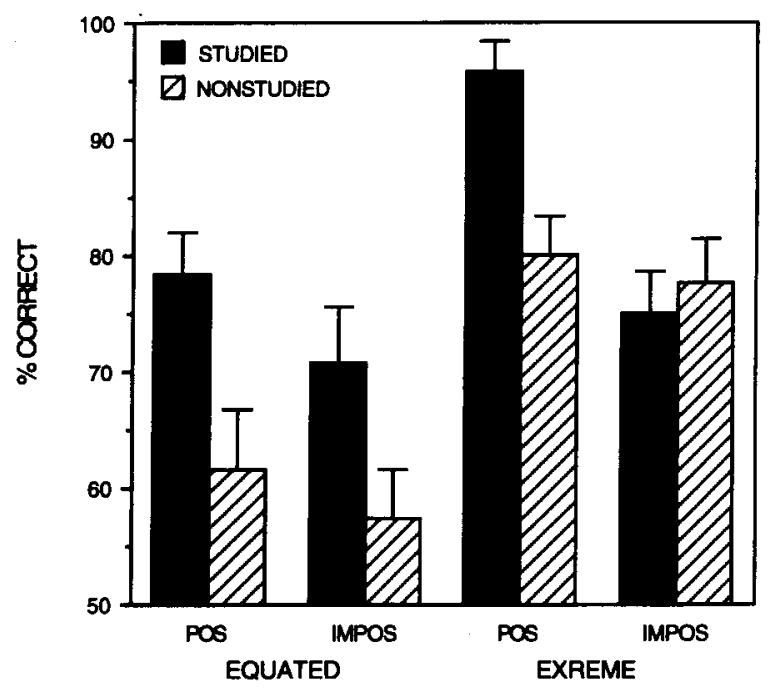

b) RECOGNITION

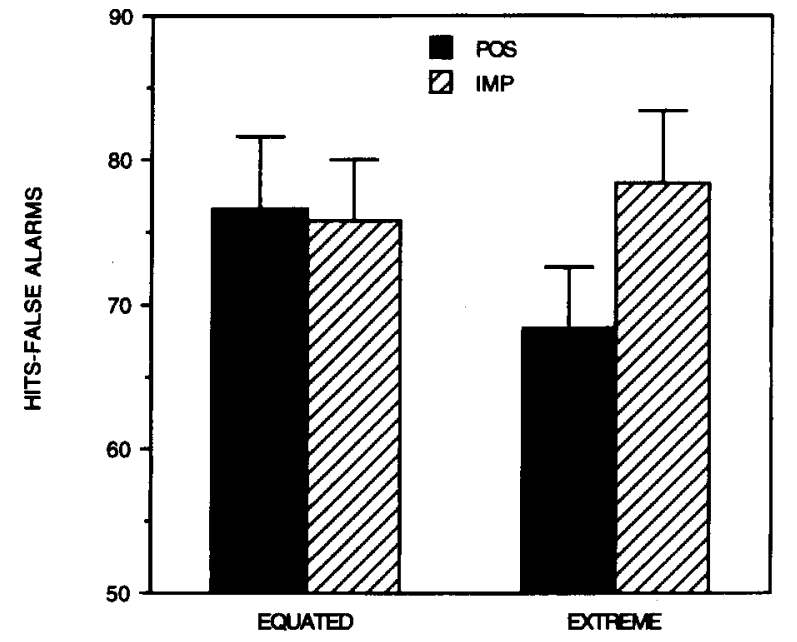

Figure 1. (a) Object decision performance when level of complexity was equated (maximally similar) or extreme (maximally dissimilar) for possible and impossible objects. (b) Recognition performance (hits - false alarms) when level of complexity was equated (maximally similar) or extreme (maximally dissimilar) for possible and impossible objects. possible objects (.35) and the difference between the extreme and the equated sets of impossible objects (.42) were higher than the difference between the equated possible and equated impossible objects (.13). This indicates that manipulating the degree of complexity within either possible or impossible objects affected the amount of priming more than did manipulating object possibilityimpossibility for a given degree of complexity.

Linear regressions, using the least squares method, were performed on the dependent variable of percent correct performance on object decision as it was regressed against the independent variable of either ratings of subjective complexity or subjective possibility-impossibility. For both studied and nonstudied items $(n=48)$, complexity accounted for $84.9 \%$ of the variance $\left(R^{2}\right)$, whereas possibility-impossibility accounted for only $68.0 \%$ of the variance. Furthermore, when both variables were considered simultaneously in a forward, stepwise regression, the accounted variance did not greatly increase: $R^{2}=87.6 \%$, adjusted $R^{2}=87.3 \%$. Likewise, for the studied items $(n=24)$, complexity accounted for $85.4 \%$ of the variance, whereas possibility-impossibility accounted for only $64.7 \%$ of the variance. Again, when both variables were considered simultaneously in a forward, stepwise regression, the accounted variance did not increase substantially: $R^{2}=88.2 \%$, adjusted $R^{2}=87.7 \%$. These regressions indicate that subjective complexity had more predictive value on performance in the object decision test than did subjective possibility-impossibility.

Taken together, the results from the complexity equated and complexity extreme conditions indicate that priming on the object decision test was observed for possible objects that were low in complexity (complexity extreme condition) and possible and impossible objects that were moderate in complexity (complexity equated conditions), but not impossible objects that were high in complexity (complexity extreme condition). Although, as mentioned earlier, there were no possible objects that were higher in complexity than impossible objects, a tentative conclusion seems warranted: object complexity, more than object possibility-impossibility, appears to be the primary determinant of priming in the object decision test.

To demonstrate the reliability of our critical finding of positive priming for impossible figures of moderate complexity, we conducted a post hoc analysis of the data that we had previously collected while pursuing research on attention and priming (Ganor-Stern et al., 1995). In that study, 40 additional participants performed the same priming task as the one reported in the present study, the primary difference being that 20 participants were shown the object figures five times at study each and the other 20 participants were shown the object figures three times each. On the basis of the subjective complexity ratings obtained in Phase 1 of the present study, we selected four possible and four impossible objects for each of the four following conditions: studied equated, nonstudied equated, studied extreme, and nonstudied extreme. Of these 32 objects, 12 matched the item type (studied vs. nonstud- 
ied objects) of the present study, 17 did not, and 3 were not used. Thus, over half of the objects that were studied in the attention and priming experiments were not studied in the present study.

These data were analyzed in the same way as reported above. The results for both the equated and the extreme complexity conditions from the present experiment were replicated. For the complexity equated condition, studied objects were classified more accurately than were nonstudied objects $[F(1,36)=10.88, p<.001$, for five exposures; $F(1,36)=15.37, p<.001$, for three exposures], there was no difference in performance for possible and impossible objects $[F(1,36)=3.64, p>.1$, for five exposures; $F(1,36)<1$, for three exposures], and there was no interaction of object and item type $(F<1$, for five and three exposures). Performance was better for studied items than for nonstudied items for both possible objects $[F(1,36)=12.3, p<.001$, for five exposures; $F(1,36)=$ $6.87, p<.001$, for three exposures] and impossible objects $[F(1,36)=5.45, p<.01$, for five exposures; $F(1,36)$ $=11.28, p<.001$, for three exposures]. In short, we again found positive priming for impossible figures with moderate levels of complexity for both three and five 1-sec exposure durations with different object figures and different participants.

Recognition memory. Schacter et al. (1990; Schacter, Cooper, Delaney, et al., 1991) analyzed their recognition data in terms of hit rates minus false-alarm rates. Given that false-alarm rates varied for possible and impossible objects in the present experiment, the recognition data were analyzed in terms of hits minus false alarms and $d^{\prime}$. From a signal detection viewpoint, hits minus false alarms is an inappropriate measure because it confounds instructional effects and the ability to discriminate studied and nonstudied stimuli (e.g., Kinchla, 1994; Murdock, 1974). However, to be consistent with the literature on this topic, the primary measure will be hits minus false alarms, but $d^{\prime}$ results will be reported where different.

Analyses indicated that recognition did not differ for possible and impossible objects in the complexity equated condition $(F<1)$, but, as shown in Figure 1b, it was marginally better for impossible objects than for possible objects in the complexity extreme condition $[F(1,38)=2.92$, $p<.1]$. The $d^{\prime}$ analysis indicates that there was an interaction of object complexity and object type $[F(1,38)=$ $4.52, p<.05$ ], and recognition was higher for impossible objects than for possible objects in the extreme condition $[F(1,38)=4.05, p<.05]$.

As with the object decision test, linear regressions were performed on the dependent variable of percent correct performance at recognition as it was regressed against the independent variable of ratings of either subjective complexity or subjective possibility-impossibility. For both studied and nonstudied items $(n=48)$, complexity accounted for $92.1 \%$ of the variance $\left(R^{2}\right)$, whereas possibility-impossibility accounted for only $77.3 \%$ of the variance. Moreover, when both variables were considered simultaneously in a forward, stepwise regression, the accounted variance did not increase substantially: $R^{2}=92.6 \%$, adjusted $R^{2}=92.3 \%$. Similarly, for the studied items $(n=24)$, complexity accounted for $93.0 \%$ of the variance, whereas possibility-impossibility accounted for only $78.4 \%$ of the variance. Again, when both variables were considered simultaneously in a forward, stepwise regression, the accounted variance did not greatly increase: $R^{2}=93.2 \%$, adjusted $R^{2}=92.9 \%$. As was the case for the object decision test, for recognition, subjective complexity had greater predictive value than did subjective possibility-impossibility.

The finding that impossible objects were recognized better than were possible objects in the extreme condition might appear inconsistent with the finding of better recognition of possible figures than of impossible figures reported previously by Schacter et al. (1990; Schacter, Cooper, Delaney, et al., 1991) for hit rates and by Seamon et al. (1995) for forced-choice recognition. However, the objective complexity measures showed that variability estimates were lower for the least complex possible figures than for the most complex impossible figures. If lower variability is associated with higher stimulus similarity, poorer recognition may occur for simple objects than for complex objects. Alternatively, if recognition is nonmonotonically related to complexity (French, 1954; Snodgrass \& Vanderwart, 1980), no simple predictions can be offered. The most salient finding from the present data is that when subjective complexity was equated, recognition for possible and impossible objects did not vary.

\section{GENERAL DISCUSSION}

This research demonstrated that Schacter and Cooper's impossible objects were subjectively more complex than their possible objects. In the object decision test, when impossible objects were high in complexity and possible objects were low in complexity, priming was found only for possible objects; when possible and impossible objects were equated at a moderate level of complexity, priming was observed for both object types. These findings indicate that previous demonstrations of a dissociation of object type and explicit and implicit memory (e.g., Schacter et al., 1990; Schacter, Cooper, Delaney, et al., 1991) may have been the result of a confound of object type and perceived stimulus complexity. Recognition and object decision priming can be observed for possible and impossible objects.

To be fair, lack of priming for impossible figures was never hypothesized by Schacter and Cooper. In fact, the first experiment reported by Schacter et al. (1990) made no prediction of an effect of object type on the recognition or object decision tests. Impossible figures were presented with possible figures "to make it plausible for participants to give either a 'possible' or an 'impossible' response on the object decision test" (Schacter et al., 1990, p. 10). The primary purpose of these experiments was to demonstrate implicit memory for unfamiliar three-dimensional objects. Lack of priming for impossible objects was given theoretical importance at the end of the paper and in subsequent papers by the suggestion that global structural "impossibility" may be difficult to represent internally (Schacter et al., 1990).

This dissociation was replicated in a lengthy series of experiments in which Schacter and Cooper found that recognition and object decision priming were also dissociated by other experimental manipulations, including object encoding conditions (Schacter et al., 1990; Schacter \& Cooper, 1993), number of exposures at study (Schacter, Cooper, Delaney, et al., 1991), object transformations of orientation or size between study and test (Cooper et al., 1992), participant differences in age (Schacter et al., 1992), and the presence of memory 
pathology (Schacter, Cooper, Tharan, \& Rubens, 1991; Schacter et al., 1993). These variables typically had large effects on recognition memory but had little or no effect on object decision priming. According to Schacter and Cooper (e.g., Schacter, 1994; Schacter et al., 1990), these dissociations reflect the output of different underlying memory systems used for the representation and retrieval of visual object information. One system, called the episodic system, is responsible for explicit memory. Because explicit memory for both possible and impossible objects has been regularly observed, this system is believed to be able to encode representations for both object types. The other system, responsible for implicit memory, is the structural description system. The primary function of this system is to analyze the structural relations among the components of a visual object to compute a global three-dimensional representation. Representations from this system are assumed to be useful during the object decision test because this test requires participants to make possible-impossible decisions about an object's three-dimensional structure. To the extent that the participants already computed these representations during study, their performance on the object decision test should be more accurate for studied objects than for nonstudied objects. The finding that priming was observed only for possible objects has been interpreted to mean that the structural description system either cannot compute or has great difficulty in computing three-dimensional representations of objects that are structurally impossible. The structural description system cannot compute "a single global interpretation of an impossible object, precisely because there is no globally consistent interpretation of the structure of such an object" (Schacter, Cooper, Delaney, et al., 1991, p. 16).

Interestingly, Schacter et al. (1990) discussed the possibility that the lack of priming for impossible objects could have been due to physical differences in the stimuli. Whereas they noted that the impossible objects were selected "to be roughly as complex and unusual as the possible objects" (p. 10), they later acknowledged that they "did not attempt to equate possible and impossible objects on such dimensions as complexity, size, or so forth" (p. 18), and they never tested them empirically. Schacter et al. (1990) did suggest that if the impossible objects were matched to the possible objects on various physical dimensions, it is conceivable that significant priming would be observed - a prediction that has been supported by the present study. On the basis of the present results, we now know that perceived object complexity is a better predictor of performance in both the object decision priming and the recognition memory tests than is object possibilityimpossibility.

Schacter and Cooper (e.g., Schacter, 1994; Schacter et al., 1990; Schacter, Cooper, Delaney, et al., 1991) have assumed that the object decision test requires access to global structural information and that the structural description system cannot compute global structural descriptions of impossible objects. However, implicit memory for impossible objects has already been demonstrated using the mere exposure paradigm. Seamon et al. (1995) presented participants with Schacter and Cooper's possible and impossible figures under various encoding conditions followed by forced-choice recognition and af fective preference tests. Performance on both tests was stochastically independent, and participants recognized and liked previously shown possible and impossible objects significantly greater than chance. Consistent with views expressed earlier by Schacter, Cooper, Delaney, et al. (1991), Seamon et al. suggested that the affective preference test allows judgments to be based on structural descriptions of possible objects or possible parts of impossible objects. Unlike the object decision test, the mere exposure test does not require global structural descriptions. The present findings support either this view or an alternative position that suggests that the structural description system is capable of computing global structural descriptions of both possible and impossible objects, providing those objects are not structurally highly complex. In either case, priming for impossible objects would be obtained. For structurally complex objects, such as the extreme impossible objects of the present study, lack of priming may be due to insufficient time and resources available for encoding. This may explain why, given subjectively complex impossible objects under the same studying conditions as the present study, Schacter and Cooper failed to observe priming for impossible objects. The demonstration of implicit memory for impossible objects in the object decision and mere exposure paradigms indicates that a reformulation of the operation of the structural description system regarding impossible objects is necessary

In conclusion, using Schacter and Cooper's stimuli and procedures, we have demonstrated for the first time that it is possible to obtain significant priming for impossible objects. Moreover, we have shown that the critical condition for this finding is perceived stimulus complexity. Specifically, impossible objects that are moderate, not high, in perceived complexity yield object decision priming. Finally, we have demonstrated that this finding is reliable by a post hoc analysis of priming data employing different participants and different stimuli from the same pool of possible and impossible objects.

\section{REFERENCES}

ATTNEAVE, F. (1955). Symmetry, information, and memory for patterns. American Journal of Psychology, 68, 209-222.

AtTnEave, F. (1957). Physical determinants of the judged complexity of shapes. Journal of Experimental Psychology, 53, 221-227.

Chipman, S. F. (1977). Complexity and structure in visual patterns. Journal of Experimental Psychology: General, 106, 269-301.

Cooper, L. A., Schacter, D. L., Ballesteros, S., \& Moore, C. (1992). Priming and recognition of transformed three-dimensional objects: Effects of size and reflection. Journal of Experimental Psychology: Learning, Memory, \& Cognition, 18, 43-57.

FEHRER, E. V. (1935). An investigation of the learning of visually perceived forms. American Journal of Psychology, 47, 187-221.

FRENCH, R. S. (1954). Identification of dot patterns from memory as a function of complexity. Journal of Experimental Psychology, 47, 22-26.

Ganor-Stern, D., Seamon, J. G., \& Carrasco, M. (June, 1995). Explicit and implicit memory for novel three-dimensional objects require attention. Poster presented at the annual convention of the American Psychological Society, New York.

GrAF, P. (1994). Explicit and implicit memory: A decade of research. In D. L. Schacter \& E. Tulving (Eds.), Memory systems 1994. Cambridge, MA: MIT Press.

GraF, P., \& SCHACTER, D. L. (1985). Implicit and explicit memory for new associations in normal and amnesic subjects. Journal of Experimental Psychology: Learning, Memory, \& Cognition, 11, 501-518.

HoCHBERG, J., \& BROOKS, V. (1960). The psychophysics of form: Reversible-perspective drawings of spatial objects. American Journal of Psychology, 73, 337-354

Kinchla, R. A. (1994). Comments on Batchelder and Reiffer's multinomial model of source monitoring. Psychological Review, 101, 166-171.

Moscovitch, M., Goshen-Gottstein, Y., \& Vriezen, E. (1994). Memory without conscious recollection: A tutorial review from a neuropsychological perspective. In C. Umiltà \& M. Moscovitch (Eds.), Attention and performance $X V$ : Conscious and nonconscious information processing (pp. 619-660). Cambridge, MA: MIT Press.

MURDOCK, B. B., JR. (1974). Human memory: Theory and data. Potomac, MD: Erlbaum.

Murphy, G. L., \& Hutchinson, J. W. (1982). Memory for forms: Common memory formats for verbal and visual stimulus presentations. Memory \& Cognition, 10, 54-61.

RatCliff, R., \& McKoon, G. (1995). Bias in the priming of object decisions. Journal of Experimental Psychology: Learning, Memory, \& Cognition, 21, 754-767.

Richardson-Klavehn, A., \& BJork, R. A. (1988). Measures of memory. Annual Review of Psychology, 39, 475-543.

Roediger, H. L., III, \& MCDERMotT, K. B. (1993). Implicit memory in normal subjects. In F. Boller \& J. Grafman (Eds.), Handbook of neuropsychology (Vol. 8, pp. 63-131). Amsterdam: Elsevier.

SCHACTER, D. L. (1987). Implicit memory: History and current status. Journal of Experimental Psychology: Learning, Memory, \& Cognition, 13, 501-518.

SCHACTER, D. L. (1994). Priming and multiple memory systems: Perceptual mechanisms of implicit memory. In D. L. Schacter \& E. Tulving (Eds.), Memory systems 1994 (pp. 233-268). Cambridge, MA: MIT Press. 
SCHACTER, D. L., \& COOPER, L. A. (1993). Implicit and explicit memory for novel visual objects: Structure and function. Journal of Experimental Psychology: Learning, Memory, \& Cognition, 19, 995-1009.

SCHACTER, D. L., \& COOPER, L. A. (1995). Bias in the priming of object decisions: Logic, assumption, and data. Journal of Experimental Psychology: Learning, Memory, \& Cognition, 21, 768-776.

Schacter, D. L., Cooper, L. A., \& Delaney, S. M. (1990). Implicit memory for unfamiliar objects depends on access to structural descriptions. Journal of Experimental Psychology: General, 119, 5-24.

Schacter, D. L., Cooper, L. A., Delaney, S. M., Peterson, M. A., \& THARAN, M. (1991). Implicit memory for possible and impossible objects: Constraints on the construction of structural descriptions. Journal of Experimental Psychology: Learning, Memory, \& Cognition, 17, 3-19.

Schacter, D. L., CoOper, L. A., Tharan, M., \& Rubens, A. B. (1991). Preserved priming of novel objects in patients with memory disorders. Journal of Cognitive Neuroscience, 3, 118-131.

Schacter, D. L., Cooper, L. A., \& Treadwell, J. (1993). Preserved priming of novel objects across size transformations in amnesic patients. Psychological Science, 4, 331-335.

SChaCter, D. L., COOPER, L. A., \& VAldiserri, M. (1992). Implicit and explicit memory for novel visual objects in older and younger adults. Psychology \& Aging, 7, 299-308.

SCHACTER, D. L., \& Tulving, E. (1994). What are the memory sys- tems of 1994 ? In D. L. Schacter \& E. Tulving (Eds.), Memory systems 1994. Cambridge, MA: MIT Press.

Seamon, J. G., Williams, P. C., Crowley, M. J., Kim, I. J., Langer, S. A., ORNE, P. J., \& Wishengrad, D. L. (1995). The mere exposure effect is based on implicit memory: Effects of stimulus type, encoding conditions, and number of exposures on recognition and af fect judgments. Journal of Experimental Psychology: Learning, Memory, \& Cognition, 21, 711-721.

SNODGRASS, J. G., \& VANDERWART, M. (1980). A standardized set of 260 pictures: Norms for name agreement, image agreement, familiarity, and visual complexity. Journal of Experimental Psychology: Learning, Memory, \& Cognition, 6, 174-215.

VAnderplas, J. M., \& Garvin, E. A. (1959). Complexity, association value, and practice as factors in shape recognition following pairedassociates training. Journal of Experimental Psychology, 57, 155-163.

\section{NOTE}

1. Subjective and objective complexity measures for the 48 objects in Phase 2 are available from the authors.

(Manuscript received August 29, 1995; revision accepted for publication January 25, 1996.) 